\title{
High-frequency electric welding: a novel method for improved immediate chorioretinal adhesion in vitreoretinal surgery
}

\author{
Nicolay Umanets • Natalya V. Pasyechnikova • \\ Vladimir A. Naumenko • Paul B. Henrich
}

Received: 2 January 2014 / Revised: 25 May 2014 / Accepted: 22 June 2014 / Published online: 17 July 2014

(C) Springer-Verlag Berlin Heidelberg 2014

\begin{abstract}
Purpose To evaluate high-frequency electric welding (HFEW) as a novel technique for retinopexy with improved immediate chorioretinal adhesion

Methods In a prospective, randomized, experimental study, we examined 104 eyes of 52 rabbits randomly assigned to either standard $810 \mathrm{~nm}$ endolaser retinopexy, alternating current 14-16 V or 18-20 V HFEW retinopexy. A full-thickness fragment of eye wall tissue containing the retinopexy was isolated $1 \mathrm{~h}, 3$ days, 1 week, or 1 month respectively after the intervention, and fixed to an analytical electronic scale. A nylon suture passed through the retina was elevated by a biomechanical force elongation tester. The reduction in weight at the time of retinopexy rupture was registered as a measure for retinopexy adhesion strength.

Results One hour post-exposure, adhesive strengths were significantly higher in both HFEW groups than in controls $(212 \pm$ $26.6 \mathrm{mg}$ and $122 \pm 16 \mathrm{mg}$ vs $104 \pm 10 \mathrm{mg} ; p=0.0001$ and $p=$ 0.024 respectively) while laser retinopexy did not significantly change adhesive strength $(114 \pm 14.0 \mathrm{mg}, p=0.149)$. Subsequent adhesive strengths were significantly increased for all retinopexy techniques: 3 days post-op 14-16 V HFEW 224 \pm $30.0 \mathrm{mg}(p=0.001), 18-20 \mathrm{~V}$ HFEW $128 \pm 15.6(p=0.001)$, laser $131 \pm 12.7 \mathrm{mg}(p=0.0007)$; at 1 week 14-16 HFEW 235 $\pm 24.7 \mathrm{mg}, 18-20$ V HFEW $213 \pm 22.4 \mathrm{mg}$, laser $188 \pm 18.7 \mathrm{mg}$ (all $p \leq 0.001$ ); 1 month post-op $14-16$ V HFEW $275 \pm$ $32.0 \mathrm{mg}, 18-20 \mathrm{~V}$ HFEW $283 \pm 31.0 \mathrm{mg}$, laser $276 \pm$ $21.7 \mathrm{mg}$, rspectively (all $\mathrm{p} \leq 0.0001$ ).
\end{abstract}

N. Umanets $\cdot$ N. V. Pasyechnikova $\cdot$ V. A. Naumenko The Filatov Institute of Eye Diseases and Tissue Therapy AMS of Ukraine, Frantsuzkyi Boulevard 49/51, Odessa 65061, Ukraine

P. B. Henrich $(\triangle)$

Department of Ophthalmology, University of Basel, P.O. Box, CH-4012, Basel, Switzerland

e-mail: paul.henrich@usb.ch
Conclusion HFEW represents a novel technique for retinopexy during vitreoretinal surgery. It allows firm chorioretinal adhesion immediately after exposure. In nonvitrectomized eyes, using $14-16 \mathrm{~V}$ is particularly effective.

Keywords Retinal detachment $\cdot$ Retinopexy $\cdot$ Electric welding $\cdot$ Laser retinopexy $\cdot$ Cryotherapy

\section{Introduction}

Chorioretinal adhesion strength is an important factor in determining the outcome of vitreoretinal surgery for rhegmatogenous retinal detachment. Retinal reattachment can only be maintained if robust closure of all retinal tears is guaranteed by firm adhesion of their circumferences to the underlying retinal pigment epithelium and choroid.

Current techniques for retinopexy during retinal detachment surgery, such as laser coagulation or cryotherapy, are not fully satisfactory, because they tend to take effect and provide reliable closure of retinal breaks only after a few weeks $[1,2]$. This results in significant morbidity from redetachment, and requires the use of vitreous tamponades. Retinopexy techniques were intensely investigated in the 1980 s, but have only infrequently been studied or technically innovated afterwards.

Retinopexy techniques with an accelerated mechanism of action are highly anticipated.

Welding is a sculptural process that joins materials by causing coalescence. Many different energy sources may be used for welding. Laser tissue welding has first been described in experimental intestinal surgery [3], while thermal energy through low-voltage direct current welding has been tested for cautery and tissue dissection in tonsillectomy $[4,5]$. Highfrequency electric welding (HFEW) has been shown recently to be a suitable technique for the colligation of biological 
tissues $[6,7]$. Based on our experimental data, we present this technique as a novel method for retinopexy with improved immediate chorioretinal adhesion.

\section{Materials and methods}

We present a prospective, randomized experimental study on 104 eyes of 52 Chinchilla rabbits of a mean age of 7 months ( \pm 1 month). Mean weight was $3.0 \mathrm{~kg}( \pm 0.5 \mathrm{~kg})$. All experimental animals were kept in standard vivarium conditions on the same diet.

Experimental retinopexies were performed without vitrectomy under general anesthesia $(1 \mathrm{ml} / \mathrm{kg}$ of intravenous sodium thiopental $10 \%$, Arterium Corporation, Kyiv, Ukraine). Animals were fixed and the operation field disinfected following a state of the art protocol (Povidone-Iodine Egis ${ }^{\mathrm{TM}}$, Egis PLC, Kyiv, Ukraine). Standard $2 \mathrm{ml}$ lidocaine retrobulbar analgesia was applied (Lidocaine Hydrochloride $2 \%$ Egis $^{\mathrm{TM}}$, Egis PLC, Kyiv, Ukraine). Pupillary dilation was achieved by instilling one drop of $1 \%$ tropicamide (Tropicamidum WZF Polfa $1 \%$, Polfa Warszawa S.A., Warsaw, Poland) into the conjunctival sac. After circular conjunctival peritomy, limbal sclerotomies were placed in the 10 and $2 \mathrm{o}^{\prime}$ clock positions in right eyes, and in the 4 and 8 o'clock position in left eyes (A-Ok ${ }^{\circledR}$ Corneal/ Scleral V-Lance, Alcon Inc., Hünenberg, Switzerland). Refractive aberrations of the leporine cornea were corrected by a flat contact lens (Disposable Vitrectomy Lens Flat 1284. DD; D.O.R.C. BV, Zuidland, The Netherlands).

Animals were randomly assigned to one of four groups:

Group "Welding one" (W1) $(N=16)$ was treated with alternating current 14-16 V HFEW: After the introduction of an endoillumination light pipe (20G Sapphire Wide Angle Illuminator, Alcon Inc., Hünenberg, Switzerland) through one sclerotomy, a $20 \mathrm{G}$ gold-tipped custom-made steel monopolar welding electrode was inserted through the remaining sclerotomy. A neutral electrode was attached to the blepharostat. The tip of the probe was brought into gentle contact with the retinal surface and the current was activated for $2 \mathrm{~s}$. Based on our previous research, the electric potential was set to $14-16 \mathrm{~V}$, while the alternating current was adjusted to $0.1 \mathrm{~A}$ at $66 \mathrm{kHz}$ [7]. Eight immediately adjacent welding applications were carried out at the level of the temporal and nasal medullary rays in two rows and at a distance of 3-4 mm from the edge of the optic disc.

Group "Welding 2" (W2) $(N=16)$ was also treated by HFEW using an identical protocol with the exception of electric potential, which was set to $18-20 \mathrm{~V}$.

In group "Laser" (L) $(N=16)$, a diode laser (Lahta, Milon Laser Co., St. Petersburg, Russian Federation) was used for retinopexy: After the introduction of the endoillumination light pipe through one sclerotomy, a laser probe (20-Gauge Straight Laser Probe, Alcon Inc., Hünenberg, Switzerland) was inserted through the remaining sclerotomy. Twelve laser applications were performed at a distance of $0.1 \mathrm{~mm}$ from one another at the level of the temporal and nasal medullary rays in two rows and at a distance of 3-4 $\mathrm{mm}$ from the edge of the optic disc. The laser probe was held at a distance of approximately $2-3 \mathrm{~mm}$ over the level of the retina. Laser parameters were adjusted to a wavelength of $810 \mathrm{~nm}$, power of 200 $300 \mathrm{~mW}$ and an exposure time of $250 \mathrm{~ms}$.

Group C $(N=4)$ served as a control and remained untreated.

After the intervention, laboratory animals were monitored for behavioral changes indicating general disease or visual disturbance. Animals were sacrificed by gas embolism within $1 \mathrm{~h}, 3$ days, 1 week and 1 month after the experiment respectively, resulting in four rabbits in each of the experimental groups and one rabbit from the control group at each point in time. Following enucleation, full-thickness fragments of eye wall tissue were isolated from the posterior pole in the form of a strip of approximately $4 \times 2 \mathrm{~mm}$ side length, consisting of sclera, choroid, and retina, in such a way that the optic disc would be centered in the middle of the fragment. The scleral surface of the fragment was fixed to the weighing platform of an analytic electronic scale (MS-P Digital scale, JR Inc., Guangdong, China). Immediately adjacent to the rim of the optic disc, the retina was incised both on the nasal and on the temporal side. A $0.5 \mathrm{~mm}$ flap of retina was lifted on each side of the optic disc. A Nylon 10-0 suture was passed through each flap at a distance of roughly $0.5 \mathrm{~mm}$ from the cutoff line (Nylon 10-0, Golnit ltd, Kyiv, Ukraine). The needle was cut off and the free end of one of the nylon threads fixed to the advancement lever of a custom-made motor-controlled biomechanical force elongation tester (Fig. 1). This device is designed to vertically pull on the end of the thread at a slow constant speed. The traction vector and speed was identical for

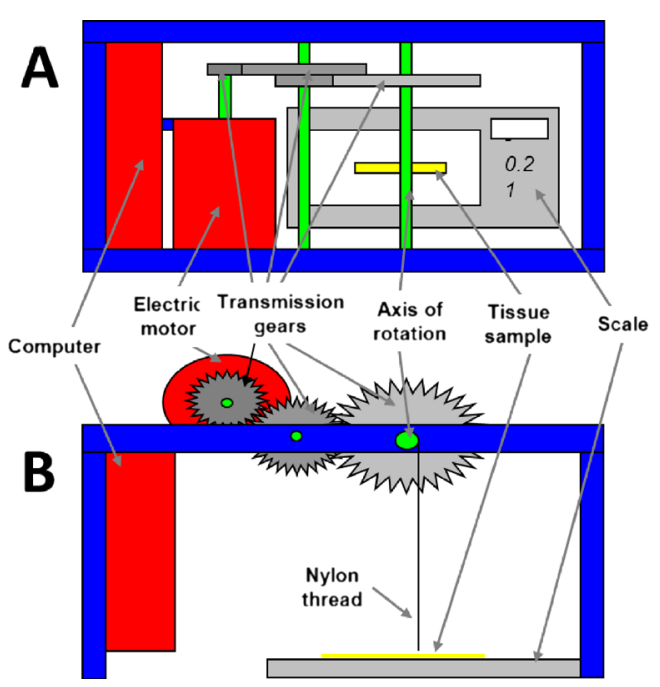

Fig. 1 Schematic drawing of the custom-made motor-controlled biomechanical force elongation tester. a Horizontal section. b Longitudinal section 
all tested groups. Pulling on the end of the nylon suture exerts traction on the retinal flap and, by this means, on the tissue specimen, which is firmly attached to the underlying weighing platform. At low vertical tractive force, gravitation of the tissue specimen is, thus, antagonized and the tissue fragment weight registered by the analytical scale decreased. Increasing vertical traction will finally result in detachment of the retina from the underlying choroid, as the chorioretinal connection represents the weakest tie in this setup. The ultimate force, defined as the force necessary to tear the tissue, is normally indicated in $\mathrm{mN}$ [8]. The ultimate force is proportional to the reduction in specimen weight registered by the scale until the moment of rupture. We, thus, used the weight change in milligram (mg) observed during the traction experiment as a measure for the strength of the chorioretinal connection. For each specimen, the experiment was performed for the nasal and the temporal retinal flap, separately.

Statistical significance levels were calculated using the Statistica 9.0 software package (StatSoft, Inc., Tulsa, OK, USA). To compare group mean values, one-way ANOVAs were performed. Results were presented as F-values of the ANOVAs with corresponding $p$-values. In cases of nonsignificance, no further comparisons of subgroups were needed. In cases of significance, a subsequent Newman-Keuls test was performed to determine the significance of mean differences. A $p$-value smaller than 0.05 was considered statistically significant for all tests in this study.

All animal experiments were conducted in accordance with the ARVO animal statement and the WMA Statement on Animal Use in Biomedical Research (as adopted by the 41st World Medical Assembly, Hong Kong, September 1989, and revised by the 57th WMA General Assembly, Pilanesberg, South Africa, October 2006). At all times, the tenets of the Declaration of Helsinki were observed.

\section{Results}

Both HFEW and laser interventions could be carried out uneventfully and without difficulty in all experimental animals. At the site of treatment, a whitish discoloration of the retina could be produced without difficulty in all 64 eyes treated with HFEW, as well as in the 32 eyes treated with laser retinopexy. All experimental animals showed prompt and uneventful recovery from the intervention and from anaesthesia, with no obvious indication of visual handicap. Measurements of chorioretinal adhesion of untreated healthy retina and choroid in the control group yielded a mean of 104 $\pm 10 \mathrm{mg}$ on the temporal side and $105 \pm 12 \mathrm{mg}$ on the nasal side of the optic disc. A $t$-test showed no statistical difference between the connective strength on both sides of the optic disc, allowing pooling of data from the nasal and temporal side $(p=0.983)$.
Analyses of specimens from the experimental groups 1 hour post-exposure showed adhesive strengths of $212 \pm$ $26.6 \mathrm{mg}$ in group $\mathrm{W} 1,122 \pm 16.1 \mathrm{mg}$ in group W2, and 114 $\pm 14.0 \mathrm{mg}$ in group L. Compared to the control group, these adhesive strengths proved to be significantly increased in groups $\mathrm{W} 1$ and $\mathrm{W} 2$ ( $p=0.0001$ and $p=0.024$ respectively), while no significant difference was found between group L and the control group ( $p=0.1493$ ) (Fig. 2a).

Three days after exposure, increased adhesive strengths were found in all experimental groups. Maximum strengths reached $224 \pm 30.0 \mathrm{mg}$ in group W1 ( $p=0.0001$ compared to the control group). Group W2 and L showed similar mean values of $128 \pm 15.6$ and $131 \pm 12.7 \mathrm{mg}$ respectively ( $p=$ 0.651 ), also both significantly superior to the control group ( $p=0.001$ and $p=0.0007$ respectively) (Fig. $2 \mathrm{~b}$ ).

One week after the operation, the strength of the chorioretinal adhesion had further increased in all experimental groups, reaching $235 \pm 24.7 \mathrm{mg}$ in group W1, $213 \pm 22.4 \mathrm{mg}$ in group W2 and $188 \pm 18.7 \mathrm{mg}$ in group L. Adhesive forces were significantly higher than in the control group in all three experimental groups $(\mathrm{p} \leq 0.0001)$ (Fig. 2c).

One month after surgery, chorioretinal adhesion strength reached a maximum in groups $\mathrm{W} 1, \mathrm{~W} 2$, and $\mathrm{L}$, with mean values of $275 \pm 32.0 \mathrm{mg}, 283 \pm 31.0 \mathrm{mg}$ and $276 \pm 21.7 \mathrm{mg}$ respectively. No significant differences between the experimental groups could be observed at this point $(p \geq 0.44)$. Adhesive forces continued to be significantly higher than in the control group in all experimental groups ( $\mathrm{p} \leq 0.0001$ for all experimental groups) (Fig. 2d).

\section{Discussion}

The principle of pars plana vitrectomy for retinal repair consists of the relief of vitreoretinal traction in combination with endodrainage of subretinal fluid. In order to secure long-term retinal reattachment, however, a reliable closure of all retinal breaks, generally referred to as retinopexy, is mandatory. Traditionally, endolaser coagulation and cryotherapy have been employed for this purpose. Retinopexy commonly requires the use of a temporary nonaqueous tamponade of the vitreous cavity, either air, fluoridated sulfur gases, or silicone oil. The rationale for using this type of tamponade is to allow sufficient time for chorioretinal adhesion to develop, while avoiding seepage of fluid through the causative break [9]. The ability of a substance to act as a tamponade therefore relies on it being immiscible with water and forming an interface with it [10]. The formation of a durable chorioretinal connection is generally completed after $2-3$ weeks $[1,2]$. The development of a chorioretinal connection is based on two complementary principles: 1) adhesion - "sticking" of the retina to the choroid immediately after treatment [11], and 2) scarring - 

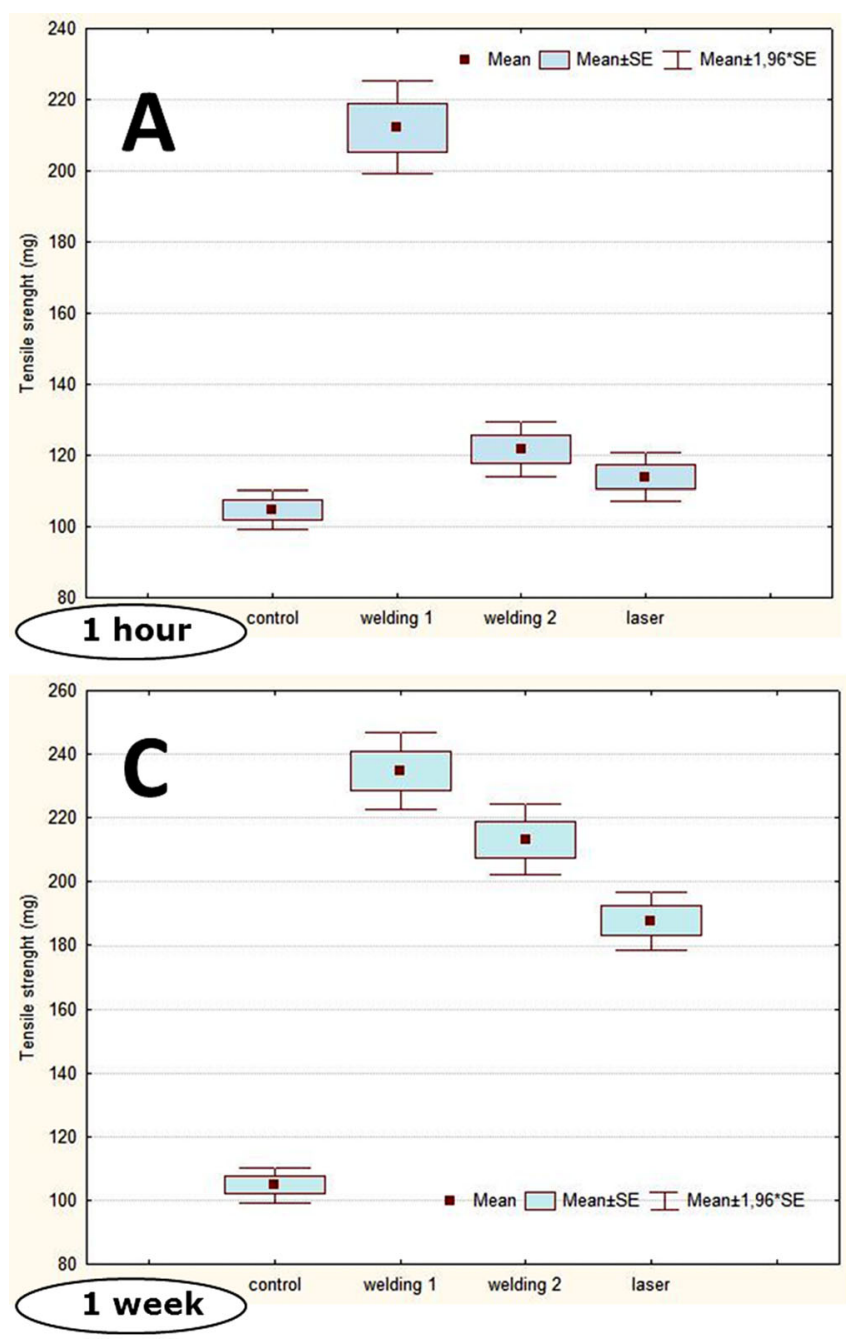

Fig. 2 Adhesive strengths $1 \mathrm{~h}$ (a), 3 days (b), 1 week (c) and 1 month (d) after retinopexy. The ordinate displays the tensile strengths in $\mathrm{mg}$. The upper and lower margins of the boxes in this standard box-and-whisker

the formation of a chorioretinal fusion scar in the affected zone [1].

Understanding of chorioretinal adhesion was initially hindered by the absence of an experimental method sensitive enough to allow accurate assessment of the changes in retinal adhesion following cryopexy [12]. Meanwhile, several methodologies have been developed to quantitatively describe tensile strength of the chorioretinal adhesion [1, 12-19]. There is consensus that the combined effects of both adhesion and scar formation result in a characteristic course of varying chorioretinal attachment strength, also termed tensile or cohesive strength, following both laser coagulation and cryotherapy, although its exact timing is still a matter of debate. An increase in cohesion to $128 \%$ of that of untreated controls during the first $24 \mathrm{~h}$, for example, has been described in an invivo rabbit model [20]. The increase was attributed to posttherapeutic adhesion which has also been described in a monkey model and one human donor eye [11]. Meanwhile,
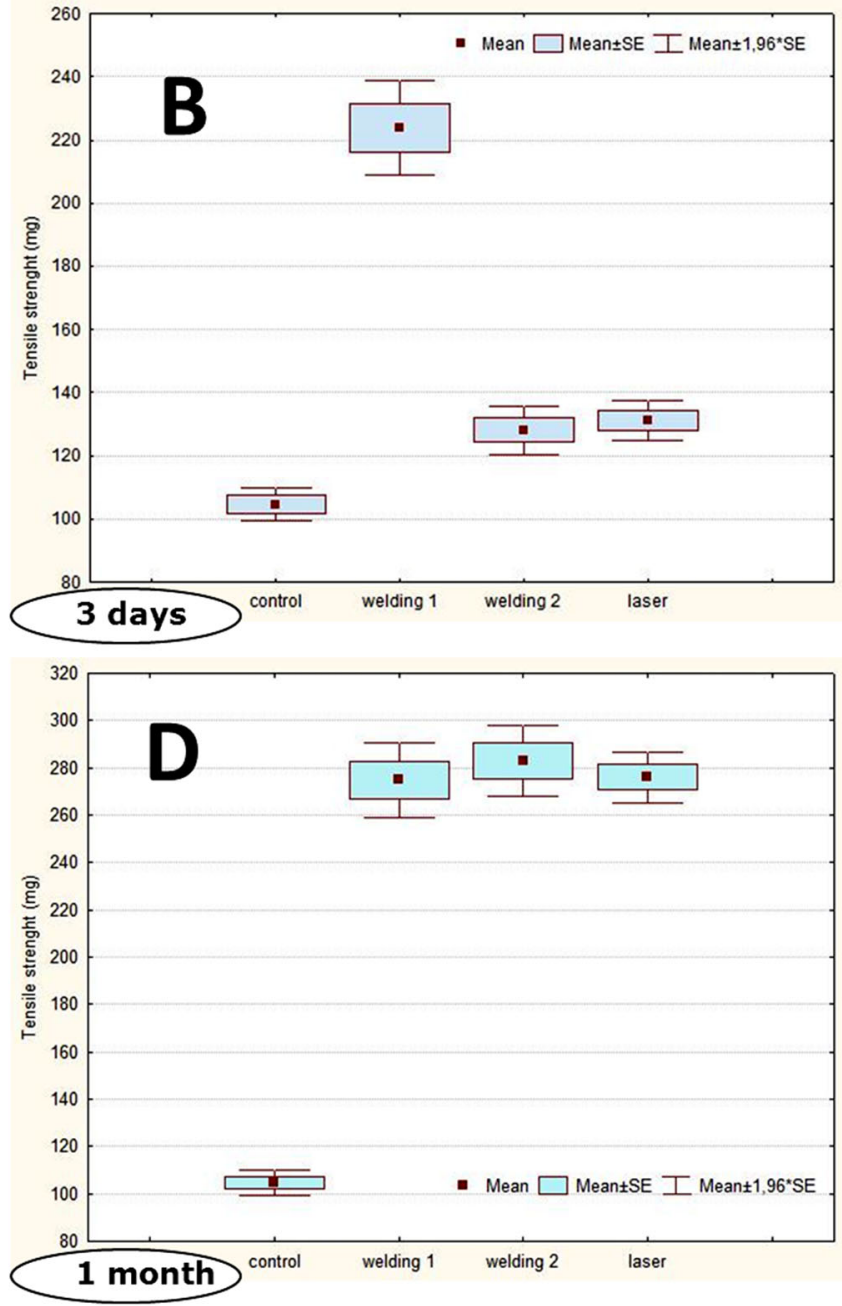

diagram represent the 25 th and the 75 th, the central line inside the box the 50th percentile (median). The whiskers mark the minimum and the maximum

another leporine study reported decreased tensile strengths immediately after laser cryopexy [19]. There is agreement, however, that tensile forces decrease during the first days after treatment: a reduction in adhesive strength during the first $24 \mathrm{~h}$ to 2 days was found by several reports, including in a feline model pilot study [1] and a rabbit model for argon laser therapy [21]. Reduced adhesive strengths within the first week were also seen for cryotherapy [20]. Reductions in tensile strengths were interpreted as a consequence of chorioretinal edema and beginning cellular infiltrations [1]. Over the following days, a gradual increase in cohesive strength was observed, reaching the level of untreated retina and choroid between the 2nd [19] and the 7th [20] day post-treatment. Over time, maximum cohesive strengths reached levels 2-3 times higher than untreated controls up to 3 weeks after exposure [1]. Long-term follow-up revealed sustained increased tensile strengths over 2 weeks to 6 months in a similar way for different retinopexy modalities. Highest values were 


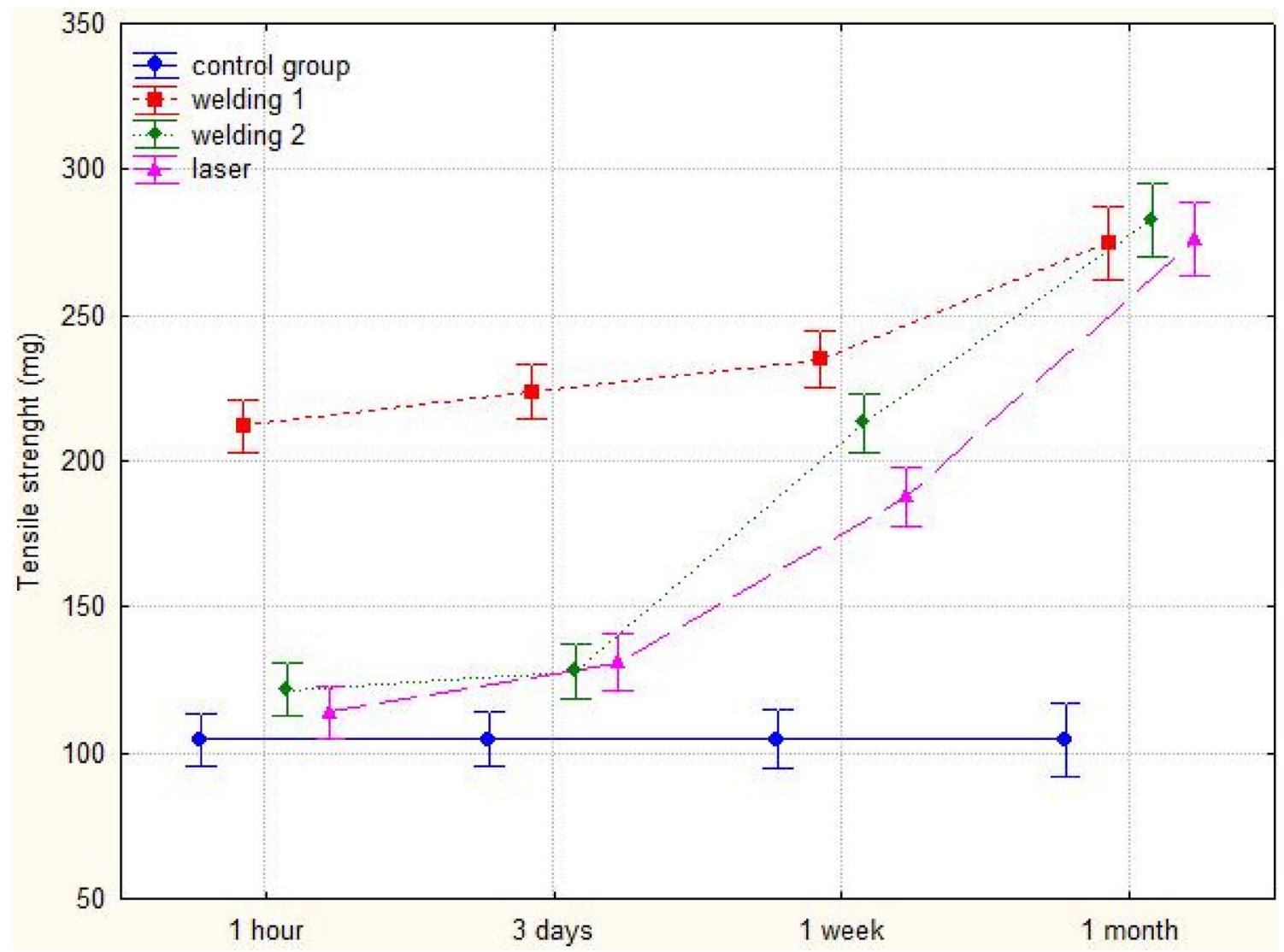

Fig. 3 Plot of adhesive strengths over the course of 1 month. Fourteen$16 \mathrm{~V}$ HFEW shows great tensile strength beginning immediately after treatment. In all other groups, initial adhesive strengths are low. A gradual increase and a convergence around on month after treatment can be

found beyond 6 months for diathermy treatment $(279 \%$ of untreated controls), cryopexy (214\%) and photocoagulation (220\%) [20]. Another study based on laser coagulation in a leporine model also yielded maximum cohesive strength of roughly 3 times that of untreated controls, although maximum strength was reached at 2 weeks post-exposure [22].

Nonetheless, the major drawback of traditional retinopexy in vitreoretinal surgery is the need for the use of vitreous tamponades. Such tamponades may be associated with morbidity from elevated intraocular pressure or cataract progression in phakic patients, and patients may experience considerable discomfort from the face-down positioning required for healing [9]. Silicone oil requires additional surgery for tamponade removal. The search for retinopexy techniques which would result in improved immediate adhesion is ongoing. It has been proposed that transscleral diode laser may result in increased amounts of absorbed thermal energy and enhance adhesion through coagulation necrosis of the affected tissue [23, 24], and a non-randomized case series showed that the ensuing adhesive forces may be sufficient to allow retinal repair without the use of vitreous tamponades in individual cases [23]. Whether adhesion is sufficient to generally permit observed. The ordinate displays the tensile strengths in $\mathrm{mg}$. The central element of each reading represents the 50th percentile (median). The whiskers mark the minimum and the maximum

reasonable protection against perioperative re-detachment is to be questioned, however [24], and in the more recent literature, the technique is not referred to any more.

HFEW has been previously shown to be a suitable technique for the colligation of biological tissues [7]. The experimental results presented in this paper show that HFEW represents an alternative retinopexy technique for vitreoretinal surgery. There are no obvious safety concerns, as the intervention was tolerated well by all laboratory animals, although objective tests for postoperative visual function, such as electrophysiological tests, were not performed. Side-effects, such as proliferative vitreoretinopathy (PVR), macular edema, inflammation, or bleeding, were not observed in any group. All experiments were performed with attached retina, and PVR formation might have been stronger if the retina had been detached. The trauma inferred from HFEW is much more localized as compared to cryotherapy, however, so that a much smaller stimulus for PVR can be expected. An ongoing clinical trial is expected to provide more solid answers with respect to clinical safety (data on file). Application is convenient and technically similar to traditional retinopexy techniques, so that no significant learning curve is to be expected 
for experienced vitreoretinal surgeons. In fact, the electrical energy used to colligate the different tissue layers is conveyed through a hand piece of similar dimensions to those of traditional laser or diathermy probes. Different from laser retinopexy and in analogy to endodiathermy, HFEW requires immediate contact between the tip of the hand piece and the treated tissue. It does not necessarily require firm adhesion between the retina and the underlying tissue, although the current experiment was performed in attached retinae. Clinical application in detached retinae also demonstrates immediate adhesion (data on file). No significant differences were found between the longterm adhesive forces measured for any of the studied retinopexy techniques at the end of the observation period (Fig. 3). Significant variations were found, however, in the short- and medium-term adhesive forces generated by the different modalities. Adhesive forces more than twice those of the control group could be achieved immediately after exposure with 14-16 V HFEW (Fig. 3). We speculate that this immediate adhesion is generated by protein denaturation: protein molecules have been previously described to be denatured and fused to one another through tissue welding, forming a tight seal [4]. Tensile strength gradually further increased throughout the first week in this group. Despite only a small difference in the intensity of the electric potential, 18-20 V HFEW yielded less solid chorioretinal connections within the first 3 days after the intervention compared to 14-16 V HFEW. One week after exposure, no significant difference was seen any more between the different HFEW modalities. These surprising effects of minor changes in voltage have been attributed to a difference in heat generation at the level of the outer retina and choroid. The range to which the tissues in question are heated depends on the settings of the electric current which passes through them. Temperatures slightly above $50-55{ }^{\circ} \mathrm{C}$, as encountered in the welding process at 14-16 V, have been described as resulting in a breakdown of outer retinal cell membranes, allowing intracellular proteins to degenerate and collect in the extracellular space, where they would blend into a substrate serving as a biological glue [25]. After welding at $18-20 \mathrm{~V}$, significantly more severe changes of the outer retina and choroid have been described, and attributed to higher temperatures, marked by coagulation necrosis of all retina layers, accompanied by partial destruction of retinal pigment epithelial cells and choroidal cells [25]. In contrast to most literature reports, a constant increase in adhesive strength with no intermittent reduction was observed for laser therapy as well as for all HFEW modalities in our sample.

The fact that both laser retinopexy and HFEW were performed in non-vitrectomized eyes may be regarded as a limitation to our study, as both treatment options would be carried out following vitrectomy in the clinical setting. Further research is needed to objectify safety and to verify whether optimum HFEW settings differ between vitrectomized and non-vitrectomized eyes.

\section{Conclusions}

High-frequency electric welding represents a novel technique for retinopexy during vitreoretinal surgery. It is technically easy to perform, and allows long-term chorioretinal connections of the same strength as conventional laser retinopexy. Its main advantage resides in a firm chorioretinal adhesion immediately after exposure. In non-vitrectomized eyes, welding at 14-16 V generates the highest immediate adhesion strengths.

Acknowledgements Statistical processing of the study data was performed by Elena Dragomiretskaya, from The Filatov Institute of Eye Diseases and Tissue Therapy AMS of Ukraine.

No payment was received from any organization, public or private, for this study. The Filatov Institute for Eye Diseases and Tissue Therapy granted permission for the use of its vivarium and the 52 examined animals, bearing all costs incurred.

Disclosures None of the authors holds any financial or proprietary interest in the material presented in this article. A patent concerning welding of the retina, but based on different parameters, is held by the Filatov Institute of Eye Diseases and Tissue Therapy:

Pat. of Ukraine № 69528 A 61 F 9/007 (25.04.2012). Method of intraoperative retinopexy for the treatment of rhegmatogenous retinal detachment. Authors: Pasyechnicova NV, Naumenko VA, Umanets NN, Zavodnaya VS, Levitskiy IM. Owner - State Institution Filatov Institute of Eye Diseases and Tissue Therapy NAMS of Ukraine.

\section{References}

1. Zauberman H (1969) Tensile strength of chorioretinal lesions produced by photocoagulation, diathermy, and cryopexy. Br J Ophthalmol 53:749-752

2. Smiddy WE, Hernandez E (1992) Histopathologic results of retinal diode laser photocoagulation in rabbit eyes. Arch Ophthalmol 110: 693-698

3. Silvola J, Salonen A, Nieminen J, Kokki H (2011) Tissue welding tonsillectomy provides an enhanced recovery compared to that after monopolar electrocautery technique in adults: a prospective randomized clinical trial. Eur Arch Otorhinolaryngol 268: 255-260. doi:10. 1007/s00405-00010-01333-00409

4. Karatzias GT, Lachanas VA, Papouliakos SM, Sandris VG (2005) Tonsillectomy using the thermal welding system. ORL J Otorhinolaryngol Relat Spec 67: 225-229

5. McCue JL, Phillips RK (1991) Sutureless intestinal anastomoses. Br J Surg 78:1291-1296

6. Zadorozhnyy O, Pasyechnikova N, Naumenko V, Lazar Y (2012) Experimental application of high-frequency electric welding of biological tissues for iridoplasty and trabeculectomy. Acta Ophthalmologica 90:0. doi:10.1111/j.1755-3768.2012.F043.x

7. Pasyechnikova N, Umanets N, Artemov A (2012) High-frequency electro-welding of the tissues of the eyeball posterior part (modified 
generator EC-300 M1) with the application of the original mono and bipolar set of instruments. Journal of Ophthalmol 2:45-49

8. Wollensak G, Spoerl E, Wirbelauer C, Pham DT (2004) Influence of indocyanine green staining on the biomechanical strength of porcine internal limiting membrane. Ophthalmologica 218:278-282

9. de Juan E Jr, McCuen B, Tiedeman J (1985) Intraocular tamponade and surface tension. Surv Ophthalmol 30:47-51

10. Hillier RJ, Stappler T, Williams RL, Turner GS, Wong D (2011) The impact of axial length on retinal tamponade for gas, silicone oil, and heavy silicone oil, using an in vitro model. Graefes Arch Clin Exp Ophthalmol 249: 671-675. doi:10.1007/s00417-00010-01579-00413

11. Folk JC, Sneed SR, Folberg R, Coonan P, Pulido JS (1989) Early retinal adhesion from laser photocoagulation. Ophthalmology 96:1523-1525

12. Zauberman H, Berman ER (1969) Measurement of adhesive forces between the sensory retina and the pigment epithelium. Exp Eye Res 8:276-283

13. DeGuillebon H, De la Tribonniere M, Pomerantzeff O (1971) Adhesion between retina and pigment epithelium. Measurement by peeling. Arch Ophthalmol 86:679-684

14. Zauberman H, de Guillebon H, Holly FJ (1972) Retinal traction in vitro. Biophysical aspects. Invest Ophthalmol 11:46-55

15. Owczarek FR, Marak GE, Pilkerton AR (1975) Retinal adhesion in light- and dark-adapted rabbits. Invest Ophthalmol 14:353-358

16. Bose M, Rassow B, Wille M (1981) The strength of retinal sear tissue after argon-, xenon-, and cryocoagulation (author's transl) Albrecht Von Graefes Arch Klin Exp Ophthalmol 216:291-299
17. Marmor MF, Abdul-Rahim AS, Cohen DS (1980) The effect of metabolic inhibitors on retinal adhesion and subretinal fluid resorption. Invest Ophthalmol Vis Sci 19:893-903

18. Kain HL (1984) A new model for examining chorioretinal adhesion experimentally. Arch Ophthalmol 102:608-611

19. Kwon OW, Kim SY (1995) Changes in adhesive force between the retina and the retinal pigment epithelium by laser photocoagulation in rabbits. Yonsei Med J 36:243-250

20. Kita M, Negi A, Kawano S, Honda Y (1991) Photothermal, cryogenic, and diathermic effects of retinal adhesive force in vivo. Retina 11:441-444

21. Kain HL (1984) Chorioretinal adhesion after argon laser photocoagulation. Arch Ophthalmol 102:612-615

22. Yoon YH, Marmor MF (1988) Rapid enhancement of retinal adhesion by laser photocoagulation. Ophthalmology 95:1385-1388

23. Martinez-Castillo V, Zapata MA, Boixadera A, Fonollosa A, GarciaArumi J (2007) Pars plana vitrectomy, laser retinopexy, and aqueous tamponade for pseudophakic rhegmatogenous retinal detachment. Ophthalmology 114: 297-302

24. Bartz-Schmidt U, Szurman P, Wong D, Kirchhof B (2008) New developments in retinal detachment surgery. Ophthalmologe 105: 27-36. doi:10.1007/s00347-00007-01662-00342

25. Umanets ND, Molchanyuk N (2013) Ultrastructural changes of the choroid and retina of a rabbit immediately after the influence of different modes of high frequency electric welding of biological tissues. Ophthalmol Journ 4:67-72 